\title{
Kluft-Matrix-Interaktion als bedeutendes Merkmal des Stofftransportes im karbonatisch gebundenen Luxemburger Sandstein
}

\author{
Claude Thielen ${ }^{1,2} \mathbb{D} \cdot$ Thomas R. Rüde ${ }^{1}$ \\ Eingegangen: 30. Juni 2021 / Überarbeitet: 1. November 2021 / Angenommen: 17. November 2021 / Online publiziert: 21. Januar 2022 \\ (c) Der/die Autor(en) 2022
}

\section{Zusammenfassung}

Der Luxemburger Sandstein ist eine kalkgebundene Sandsteinfazies, die sich während des frühen Jura auf einem flachmarinen Schelf abgelagert hat. Mit seinem ausgezeichneten Porenanteil bildet er das wichtigste Grundwasservorkommen Luxemburgs, besitzt durch seine Klüfte und deren Bedeutung für den Grundwassertransport jedoch einen komplexen Stofftransport. Diese Arbeit untersucht mithilfe von Laborexperimenten die Kluft-Matrix-Interaktion und ihre Auswirkung auf den Stofftransport im oberflächennahen Aquifer. An Bohrkernen und frisch gebrochenem Material aus einem Steinbruch werden Matrixparameter wie Porenvolumen, effektiver Diffusionskoeffizient, Durchlässigkeitsbeiwert und Porendurchmesser ermittelt. Der Stofftransport und die Interaktion der Matrix mit den Klüften als vorrangigem Transportweg werden in einem Einzelkluftversuch im Labormaßstab mit Tracerversuchen untersucht. Der Stoffaustrag lässt sich auf die Rückdiffusion aus der Matrix in das Kluftwasser, die Durchströmung poröser Matrix-Kluft-Randzonen sowie der Kluft selbst zurückführen.

Schlüsselwörter Einzelkluftversuch $\cdot$ Kluft-Matrix-Interaktion $\cdot$ Sandstein $\cdot$ Luxemburg

\section{Fracture-matrix interaction as a significant feature of mass transport in carbonate-bound luxembourg sandstone}

\begin{abstract}
The Luxembourg Sandstone is a calcareous sandstone formation deposited during the Early Jurassic on a shallow marine shelf. Due to its excellent pore content, it forms the most important groundwater reservoir of Luxembourg. However, it has a complex mass transport due to its fractures and their importance for groundwater transport. This work uses laboratory experiments to investigate the fracture-matrix interaction and its effect on solute transport in the near-surface aquifer. Matrix parameters such as pore volume, effective diffusion coefficient, permeability coefficient, and pore diameter are determined on drill cores and freshly fractured material from a quarry. Mass transport and the interaction of the matrix with the fractures as the primary transport pathway are investigated in a laboratory-scale single fracture test using tracer tests. Mass discharge can be attributed to back-diffusion from the matrix into the fracture water, flow through porous matrix-fracture boundary zones, and the fracture itself.
\end{abstract}

Keywords Single fracture experiment $\cdot$ Fracture-matrix interaction $\cdot$ Sandstone $\cdot$ Luxembourg

Claude Thielen

claude.thielen@rwth-aachen.de

1 Hydrogeologie, RWTH Aachen, Lochnerstr. 4-20, 52064 Aachen, Deutschland

2 Administration de la gestion de l'eau, avenue du Rock'n'Roll 1, 4361 Esch-sur-Alzette, Luxemburg

\section{Einleitung und Zielsetzung}

Die Trinkwasserversorgung Luxemburgs ist in großem MaBe abhängig von der Bewirtschaftung der Grundwasserreserven. In etwa $50 \%$ des genutzten Rohwassers stammt von Brunnen oder Quellanlagen, welche den Luxemburger Sandstein nutzen (Colbach 2005; AGE 2013, 2021). Der Luxemburger Sandstein ist eine diachrone lithostratigraphi- 
sche Einheit, die vor 200-196 Ma auf einem flachmarinen Schelf subtidal abgelagert worden ist (Dittrich 1984; Colbach 2005). Ein markantes Merkmal ist der karbonatische Zement, der aus Bioklasten entstanden ist. Er hat eine bimodale Verteilung mit Gehalten von 10-20\% und 35-60\% und kann in extremen Fällen bis zu $90 \%$ betragen (Colbach 2005; Kausch und Maquil 2018). Der Luxemburger Sandstein ist dann mit einem sehr dichten Kalkstein vergleichbar, wodurch es zu sehr geringdurchlässigen Gesteinsabschnitten kommen kann. Die mittlere effektive Porosität der Gesteinseinheit liegt bei $10 \%$ wobei etwa $1 \%$ auf die Klüfte entfällt, welche die Hauptwegsamkeiten des Grundwassers darstellen (Colbach 2005). Die Klüfte besitzen zwei Vorzugsrichtungen, die aufgrund der tektonischen Geschichte hauptsächlich in die Richtungen SW-NE und NW-SE streichen und abschnittsweise nahezu im rechten Winkel zueinander liegen können (Schubert 2015; Thielen 2017). Hydrogeologisch und wasserwirtschaftlich ist der seichte Luxemburger Sandstein im zentralen Luxemburg mit zahlreichen Quellaustritten und Quellfassungen vom tiefen, gespannten und nur durch Bohrungen und Brunnen erschlossenen Abschnitt im SW des Landes zu unterscheiden. Erster ist Gegenstand unserer Untersuchungen.

Die Natur des Aquifers erschwert die korrekte Eingrenzung von Schutzzonen sowie die Lokalisierung und etwaige Beseitigung von Grundwassergefährdungen und insbesondere ubiquitären Stoffeinträgen. Aus diesem Grund werden Tracerversuche genutzt, um die Einzugsgebiete der Grundwassergewinnungsanlagen zu bestimmen. Dabei sind häufig sehr geringe Wiederfindungsraten festzustellen. Meus und Willems (2021) berichten von einer durchschnittlichen Wiederfindungsrate von immerhin $26 \%$. Dieser Wert ist nach eigenen Versuchen sehr hoch, und zum Teil beträgt die Wiederfindung nur wenige Promille. Bei einer beispielhaften Untersuchung wurden über zwei Versickerungsbereichen auf der Hochfläche des Luxemburger Sandsteins die ein bis drei Meter mächtigen Bodenschichten abgetragen und offene Klüfte freigelegt, in die Farbtracer (2 kg Uranin und $6 \mathrm{~kg}$ Eosin) mit Nachspülungen von 1 bzw. $3 \mathrm{~m}^{3}$ eingeleitet wurden. Am von den Einleitorten 350-800 m entfernten Beprobungsort - eine Quellstube - traten die Tracer nach ca. $14 \mathrm{~h}$ erstmals mit Maximalgeschwindigkeiten von 23 bzw. $60 \mathrm{~m} \mathrm{~h}^{-1}$ auf. Der weitere, über rund 5 Monate erfasste Traceraustrag war an Regenereignisse gebunden, und insgesamt wurde weniger als ein Promille der Eingabemassen wiedererhalten. Ähnliches wurde auch für den Transport von Mikroorganismen beobachtet.

Für diesen periodenhaften Transport wird vermutet, dass beim direkten Einbringen eines Stoffes in den Aquifer dieser aufgrund der hohen Porosität zum großen Anteil in den Porenraum der Matrix gelangt. Über die Klüfte erfolgt mit hohen Geschwindigkeiten von zehner Metern pro Stunde ein erstes, schnelles Ausspülen der kluftgebunde- nen Stoffanteile und anschließende Abnahme der messbaren Konzentrationen an Quellaustritten. Die durch den variierenden Kalkgehalt weit streuende Matrixporosität sowie die Kluftverteilung führen zu einem ungleichmäßigen Stofftransport und sowohl zu räumlichen als auch zeitlichen Abschnitten, in denen eine Rückdiffusion aus der Matrix in stoffentleerte Klüfte stattfindet. Bei einem Regenereignis werden diese Bereiche durchspült und ein Austrag kann gemessen werden, welcher bei kontinuierlichem Durchfließen möglicherweise unter dem Messbereich liegt. Dieser Prozess wiederholt sich so lange bis die Matrix keinen Konzentrationsunterschied mehr mit dem eindringenden Versickerungswasser aufzeigt.

Ansätze für die Strömungs- und Transportmodellierung in geklüftetem Gestein sind zahlreich veröffentlicht worden (z.B. Berre et al. (2019); de Borst (2017); Karay und Hajnal (2015); Tang et al. (1981)), jedoch wird dort wenig auf die Diffusion als einer der Treiber des Stofftransportes eingegangen. Zusätzlich wird Diffusion zumeist als gesonderter Matrixparameter im Labor ermittelt (Bodin et al. 2003) und nicht im Zusammenhang eines kombinierten Versuches. Nach Haddad et al. (2012) ist die Größe der Versuchskörper bei der Diffusion ein entscheidender Einflussfaktor, wodurch sogar jene Experimente, welche die Diffusion berücksichtigen, mit den genutzten Kernproben aufgrund der Randbedingungen an Grenzen der Vergleichbarkeit zu den realen Bedingungen stoßen.

Um diesen Prozess besser zu verstehen und in künftigen numerischen Modellierungen einfließen zu lassen, wurden anhand zahlreicher Untersuchungen am Probengestein sowie einem Versuch an einer Einzelkluft die natürlichen Verhältnisse im Labormaßstab nachgestellt und ausgewertet.

\section{Methodik}

Die Labor- und Auswertungsarbeiten fanden am Lehrstuhl für Ingenieur- und Hydrogeologie der RWTH Aachen University statt. Die Laborversuche wurden an einer, für den seichten Luxemburger Sandstein repräsentativen Gesteinsprobe durchgeführt, welche eine gute Festigkeit und gleichzeitig eine gute Porosität besitzt. Die Eigenschaften dieses Gesteines werden detailliert untersucht, um das Zusammenspiel zwischen Matrix und Kluft besser zu verstehen. Ebenfalls sollen diese ermittelten Parameter in zukünftige Arbeiten bei der Modellierung des Stofftransportes zwischen Matrix und Kluft einfließen.

\section{Materialwahl}

Das für die Versuche ausgesuchte Gestein stammt aus einem Steinbruch nahe der Ortschaft Ernzen (Zentral Luxemburg) und ist repräsentativ für den seichten Luxemburger 
Sandstein. In tieferen, anoxischen Bereichen besitzt der Sandstein eine bläuliche Färbung und einen nicht quantifizierten Pyritgehalt (Colbach 2005), wohingegen das genutzte Gestein und der betrachtete Bereich bereits einer stark vorangeschrittene Pyritverwitterung unterliegen. Das Gestein besitzt entsprechend eine gelblich-weiße Farbe.

Die Kornverteilung ist gleichmäßig mit einer durchschnittlichen Korngröße von circa 0,2 bis $0,4 \mathrm{~mm}$, ohne jegliche Einschlüsse oder Hohlräume. Aus einer Charge an Gestein wurde ein Block mit den Maßen $30 \times 30 \times 20 \mathrm{~cm}$ zugeschnitten und an der kurzen Achse, entlang der natürlichen Schichtung, in der Mitte gebrochen. Die so entstandene Kluft hat pro Gesteinshälfte eine planare Kontaktfläche von $900 \mathrm{~cm}^{2}$. Ebenfalls ist die mögliche Eindringtiefe an jedem Punkt der Kluftoberfläche identisch, sodass Randeffekte im gleichen Maße auftreten und, unabhängig der präferenziellen Fließwege innerhalb der Kluft, alle Flächen den gleichen Bedingungen unterliegen. Im Vergleich $\mathrm{zu}$ Bohrkernproben liegt somit eine wesentlich größere Kontaktfläche und ein größeres Matrixvolumen vor, sodass eine bessere Annäherung an die Geländesituation besteht und zugleich die Vorteile von gut einstellbaren und dokumentierbaren Laborexperimenten bewahrt bleiben. Aus weiteren Abschnitten der gewählten Charge wurden Kerne ausgebohrt, welche für die Bestimmung der Matrixparameter genutzt wurden.

Eine besondere Herausforderung für hydraulische Versuche am Luxemburger Sandstein ist die hohe Löslichkeit des calcitischen Zements, die Versuchsergebnisse stark beeinflussen kann. Um der Lösung des calcitischen Zements entgegenzuwirken, wurde ein synthetisches Grundwasser verwendet, welches sich rechnerisch im Kalk-KohlensäureGleichgewicht befindet. Es lagen diverse Grundwasserzusammensetzungen aus Quellen und Bohrungen vor, welche jedoch stark von der jeweiligen örtlichen Situation abhängig sind und nur bedingt als Grundlage für eine eigene Mischung verwendet werden können. Daher wurde vor den eigenen Versuchen Gestein der verwendeten Charge in Wasser gelagert und bei Labortemperatur über mehrere Monate ruhen gelassen. Unter der Annahme, dass nach dieser Zeitspanne keine Reaktion zwischen Wasser und Gestein mehr auftritt und das Kalk-Kohlensäure-Gleichgewicht erreicht ist, wurde das Wasser analysiert und diese Werte als Ausgangszusammensetzung für die Berechnung eines synthetischen Wassers mit PhreeqC verwendet. Das synthetische Grundwasser wurde dann aus Reinstwasser und der berechneten Menge von Calciumchlorid-Dihydrat und Natriumhydrogencarbonat hergestellt. Weitere Ionen wurden nicht hinzugefügt, da diese für das Kalk-KohlensäureGleichgewicht keine oder eine sehr untergeordnete Rolle spielen.

Um dem Wachstum von Mikroorganismen entgegenzuwirken, wurde das Gestein vor dem Einbau in den jewei- ligen Versuchsaufbau über mehrere Tage in einem Trockenofen bei $80^{\circ} \mathrm{C}$ gelagert. Eine höhere Temperatur wurde vermieden, da nicht auszuschließen ist, dass bei der Verdampfung des eingeschlossenen Wassers das Gestein Risse bekommen würde. Zusätzlich wurde beim Einzelkluftversuch eine UV-Lampe im Zulauf platziert, um das Wasser vor Eintritt in den Versuch zu sterilisieren. Die Versuchszeiten wurden so geplant, dass einer Bildung von Mikroorganismen durch lange Standzeiten entgegengewirkt wurde. Bakterizide, wie z.B. Natriumazid, beeinflussten bei Testläufen den pH-Wert des Wassers erheblich, wodurch das Kalk-Kohlensäure Gleichgewicht verändert wurde, oder erbrachten nur kurzzeitig eine wachstumshemmende Wirkung. Auf den Einsatz solcher Stoffe wurde daher bei den späteren Untersuchungen vollständig verzichtet. Um einer vorzeitigen Bildung von Biofilmen auf der Gesteinsoberfläche entgegenzuwirken, wurden die Gesteinshälften und Kerne nicht im Versuchsaufbau, sondern in einer Unterdruckkammer im Vorfeld des Einbaus gesättigt.

Als Tracerstoff wurde Bromid verwendet, da dieser chemisch, biologisch und zeitlich stabil ist (Leibundgut et al. 2009), leicht messbar ist und im Gegensatz zu z. B. Uranin nicht von Mikroorganismen verwertet werden kann. AuBerdem wird Bromid nicht durch UV-Strahlung zerstört, wodurch das Versuchswasser ohne Problem an der oben genannten UV-Lampe vorbeigeführt werden kann. Hydratisiertes Bromid hat einen durchschnittlichen Durchmesser von $0,66 \mathrm{~nm}$ für die erste hydratisierte Schicht (D'Angelo et al. 2010). Es ist damit nur wenig größer als das Wassermolekül und klein genug für den Transport in den Poren des Sandsteins (s. unten). Entsprechend beziehen sich alle ermittelten, für den Stofftransport relevanten Parameter auf den genutzten Bromidtracer.

\section{Bestimmung von Gesteinseigenschaften}

Der Kalkgehalt des Gesteins wurde mittels gasometrischer Messung an einer Scheibler Apparatur nach DIN 18129 analysiert. Dazu wurden Bruchstücke in einem Mörser zerkleinert, bis sich ein einheitliches Pulver ergab. Dieses Pulver wurde anschließend für die Karbonatbestimmung genutzt.

Zur Ermittlung der Porosität wurden Versuchskerne mittels Helium-Gaspyknometrie und der archimedischen Dichtebestimmung untersucht. Beide Versuche ermitteln die reine Matrixdichte und über die Differenz zur Gesamtdichte kann der Anteil des Hohlraumes bestimmt werden.

Porendurchmesser wurden mittels Wicking-Versuch an einem Tensiometer durch die Gewichtszunahme eines Versuchskerns, welcher mit einem Ende in Cyclohexan eingetaucht wurde, über die Zeit ermittelt. Stellt man die Gleichung nach Weber und Stanjek (2012) nach dem Poren- 
Abb. 1 Aufbau des Diffusionszellenversuches. Die Mantelfläche des zylindrischen Versuchskörpers ist umströmungsdicht eingebaut. Durch identische Förderung wird auf beiden Seiten der Diffusionszelle eine kontinuierliche Messung der Konzentrationsänderung möglich, ohne eine Strömung in der Probe zu induzieren. Die Bromidkonzentrationen werden mit ionenselektiven Elektroden kontinuierlich erfasst

Fig. 1 Setup of the diffusion cell experiment. The circumferential surface of the cylindrical test body is installed so as to be impermeable to flow. Identical conveying on both sides of the diffusion cell allows continuous measurement of the concentration change without inducing flow in the sample. The bromide concentrations are continuously recorded with ion-selective electrodes

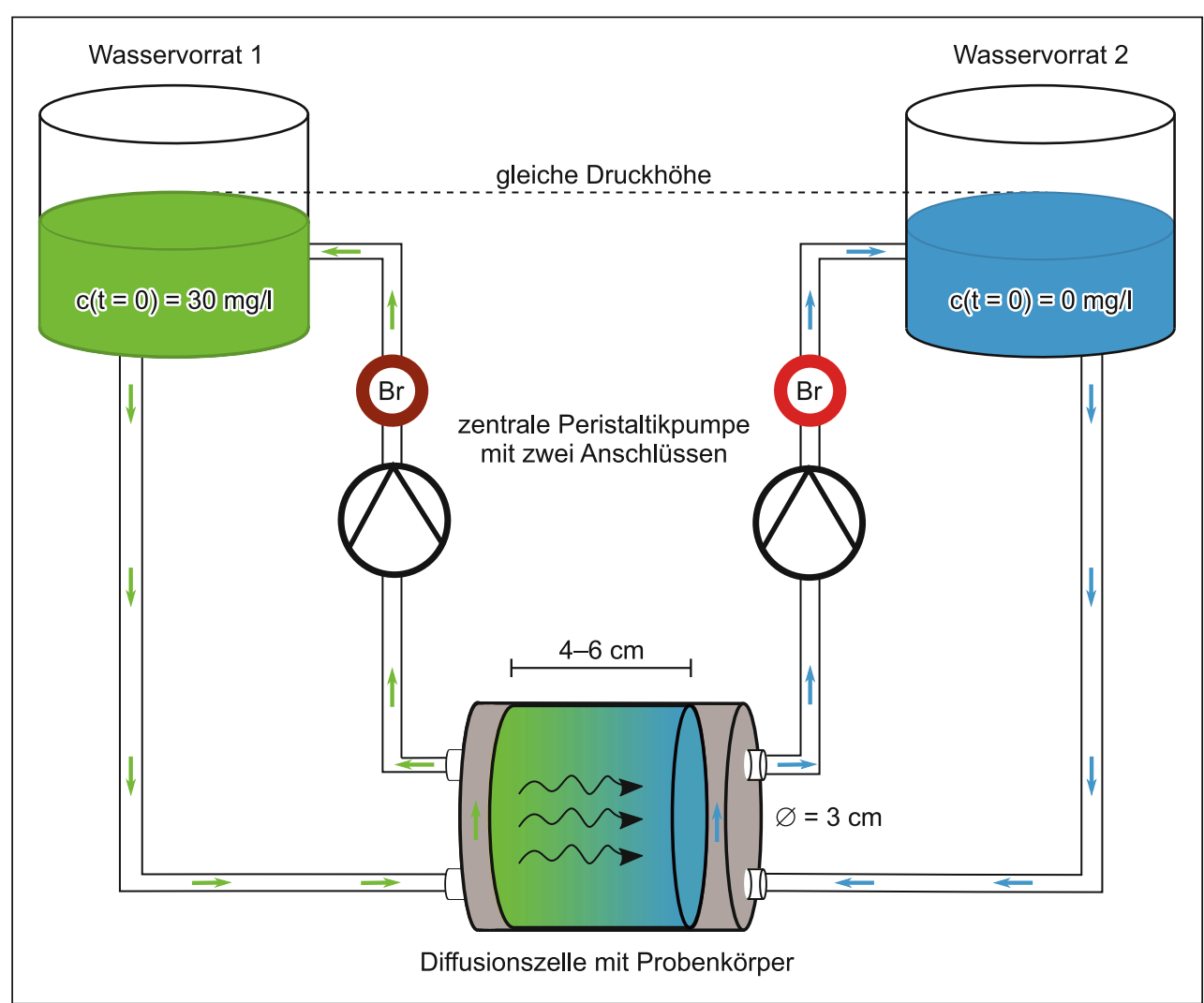

durchmesser um, erhält man die folgende Gleichung für die Bestimmung des Porenradius:

$r=S \cdot \frac{2 \eta}{\phi_{\mathrm{eff}}^{2} \cdot A^{2} \cdot \rho^{2} \cdot \sigma_{l v} \cdot \cos \theta}$

Der Porendurchmesser r [m] entspricht der Geradensteigung $\mathrm{S}\left[\mathrm{g}^{2} / \mathrm{s}\right]$ der Verlaufskurve der Gewichtszunahme gegen die Versuchszeit, multipliziert mit der doppelten dynamischen Viskosität der Flüssigkeit $\eta[\mathrm{g} /(\mathrm{m} \cdot \mathrm{s})]$ und geteilt durch das Produkt der quadrierten effektiven Porosität $\phi_{\text {eff }}[-]$ mit der quadrierten Kontaktfläche $\mathrm{A}\left[\mathrm{m}^{2}\right]$, der quadrierten Dichte der Flüssigkeit $\rho\left[\mathrm{g} / \mathrm{m}^{3}\right]$, der Oberflächenspannung der Flüssigkeit $\sigma_{\mathrm{lv}}\left[\mathrm{g} / \mathrm{s}^{2}\right]$ und des Cosinus des Kontaktwinkels $\theta$ [Deg]. Der Kontaktwinkel bei Cyclohexan kann mit $0^{\circ}$ angenommen werden. Elektrisch-osmotische Eigenschaften können aufgrund der in den Proben fehlenden Tonminerale vernachlässigt werden.

Der hydraulische Durchlässigkeitsbeiwert (nach Langguth und Voigt 2004; DIN 4049) der Gesteinsmatrix wurde nach DIN EN ISO 17892-11 in einer Triaxialzelle mit isotroper statischer Druckbeaufschlagung und konstanten Druckhöhen bestimmt. Das entgaste Oberwasser wurde in das errechnete Kalk-Kohlensäure-Gleichgewicht gesetzt. Um der Lösungsempfindlichkeit des calcitischen Zements entgegenzuwirken, wurden zudem nur geringe Druckgradienten verwendet. Der Auslauf der Zelle wurde mit einem kalibrierten Tropfenzähler gemessen. Der Versuch wurde am selben Kern mehrmals für verschiedene Gradienten durchgeführt.

\section{Bestimmung des effektiven Diffusionskoeffizienten}

Zur Bestimmung des effektiven Diffusionskoeffizienten der porösen Gesteinsmatrix wurde die in Abb. 1 schematisch dargestellte Diffusionszelle genutzt. Die kleinen Wasservolumen von ca. $10 \mathrm{ml}$ der Zellen sind unmittelbar an Vorratsbehälter (je $150 \mathrm{ml}$ ) mit gleicher Druckhöhe angeschlossen. Die kleinen Volumen im direkten Gesteinskontakt lassen sich schwer beproben, wohingegen die großen Volumen träge auf Änderungen reagieren. Aus diesem Grund wurde hier beides kombiniert und ein Kreislaufsystem eingesetzt. Durch die Verwendung einer einzelnen Peristaltikpumpe, welche zwei Schläuche aufnehmen kann, wurde über die Versuchszeit ein kontinuierlicher Volumenaustausch zwischen Zelle und Vorrat auf beiden Seiten des Versuchskerns ermöglicht. Durch die deutlichen Konzentrationsunterschiede der Bromidkonzentration in den beiden Vorratsbehältern $(0 \mathrm{mg} / \mathrm{l}$, resp. $30 \mathrm{mg} / \mathrm{l})$, kommt es zu einer Diffusion durch die mit synthetischem Versuchswasser (s. oben) vorgesättigte Kernprobe. Die Konzentrationsänderungen über die Zeit wurden mittels ionenselektiver Elektroden an den Ausgängen auf beiden Kernseiten gemessen. 
Abb. 2 Aufbau des Einzelkluftversuchs (Schema oben, Ansicht unten) mit einstellbarer Druckhöhe im Zulauf links, der Einzelkluft des Versuchskörpers ( 6 ) und Messeinrichtungen im Ablauf rechts. Erläuterung der Ziffern siehe Text

Fig. 2 Design of the single jont test (diagram above, view below) with adjustable pressure head in the inlet on the left, the single joint of the test body ( 6 ) and instrumentation in the outlet on the right. See text for explanation of the figures

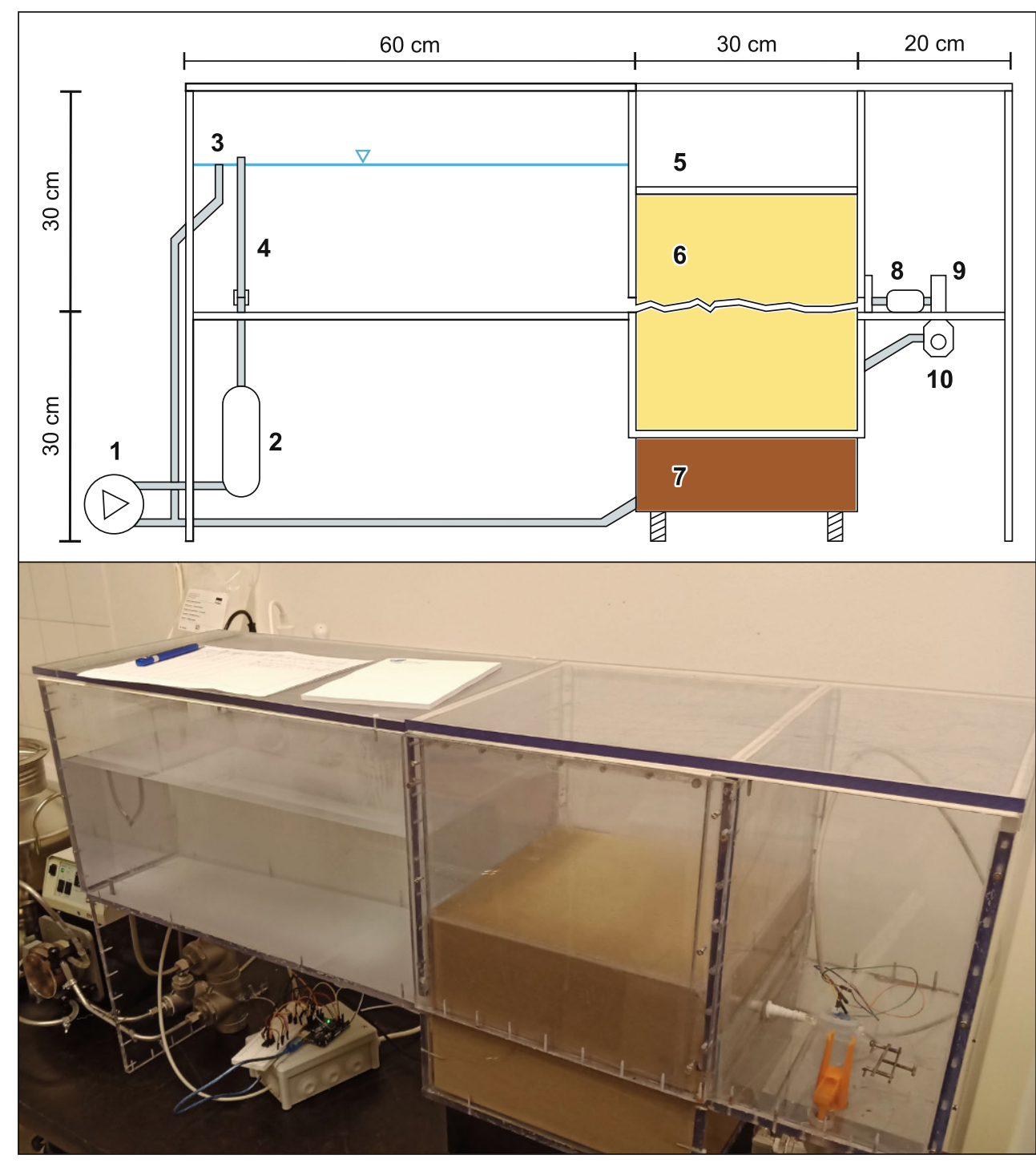

Es wurden insgesamt drei Kerne untersucht, welche aus einem Gesteinsblock in drei Raumrichtungen ausgebohrt wurden. Neben dem effektiven Diffusionskoeffizienten wurde so ebenfalls die Isotropie des Gesteins untersucht. Mit der Gleichung nach Feenstra et al. (1984) wurde der effektive Diffusionskoeffizient anhand der Konzentrationsveränderung bestimmt.

$D_{\left(t_{1} \rightarrow t_{2}\right)}^{\prime}=\frac{\left(c_{t_{2}}^{B}-c_{t_{1}}^{B}\right) \cdot L \cdot V^{B}}{A \cdot c^{A} \cdot\left(t_{2}-t_{1}\right)}$

Dabei handelt es sich in der Gleichung bei $\mathrm{D}^{{ }^{\prime}(11 \rightarrow 12)} \mathrm{um}$ den effektiven Diffusionskoeffizienten $\left[\mathrm{m}^{2} / \mathrm{s}\right]$, bei c um die Konzentration im Vorrat mit (A) und ohne (B) Bromid $[\mathrm{mg} / \mathrm{l}], \mathrm{L}[\mathrm{m}]$ ist die Länge des Probenkörpers, $\mathrm{V}^{\mathrm{B}}\left[\mathrm{m}^{3}\right]$ das Wasservolumen im bromidfreien Vorrat, A $\left[\mathrm{m}^{2}\right]$ die Kontaktfläche mit dem Probenkörper und $\mathrm{t}$ [s] die End- bzw. Startzeit des betrachteten Zeitraumes.
Die Versuchslösungen werden auf beiden Seiten des Probenkörpers im Kreislauf gefördert, wobei das Volumen über die Versuchsdauer unverändert bleibt, da aufgrund der Messmethode keine Proben genommen werden müssen. Bei Einstellen einer konstanten Steigung der Konzentrationsganglinie auf der Zielseite der Diffusion (B, in Abb. 1 rechts) kann die oben genannte Gleichung angewendet werden.

\section{Einzelkluftexperimente}

Der für das Einzelkluftexperiment verwendete Aufbau ist in Abb. 2 skizziert. Der Vorlaufbehälter (links in Abb. 2) wird über eine Peristaltikpumpe (1) befüllt. Vor dem Einlauf wird das Wasser mit einer UV-Lampe (2) sterilisiert. Über den Überlauf (3) und den Einlauf (4) wird die Füllhöhe und damit der hydraulische Gradient eingestellt. Das Gestein mit der Kluft (6) wird mit einem höhenverstell- 
baren Sockel (7) auf Höhe des Vorlaufs gebracht und mit einer Abdeckplatte (5) verschlossen, um auf allen Seiten die gleichen Randbedingungen zu schaffen. Zur Entlüftung besitzt die Abdeckplatte ein Ventil was verschlossen wird, sobald das Versuchswasser keine Luft mehr aus dem Gestein verdrängt, was bei den vorgesättigten Proben nach wenigen Minuten eintritt. Der Durchfluss wird mittels eines elektrischen Durchflusszählers (8) erfasst und kann mit einem verstellbaren Auslauf (9) über den Gradienten angepasst werden. Eine Bromidmessung mittels ionenselektiver Sonde wird über Durchflussgefäße zwischen den Punkten 8 und 9 durchgeführt. An einem Probenhahn (10) können zudem bei Bedarf Wasserproben entnommen werden.

Das Prinzip ähnelt diversen anderen Aufbauten, in welchen Kerne untersucht wurden, wie zum Beispiel Rose (2010) oder Weede und Hötzl (2005). Der hier vorgestellte Versuchsaufbau basiert auf einem Vorgängermodell von Reisinger (2020), wurde aber deutlich weiterentwickelt. Bei dem beschriebenen Aufbau wird zwischen hydraulischer und hydrochemischer Untersuchung unterschieden. Bei ersterem wird die gesamte Länge des Vorratsabteils genutzt und bis zur Höhe des Überlaufs mit Versuchswasser gefüllt. Über den einstellbaren Gradienten am Auslauf können leichte Abweichungen zwischen unterschiedlichen Versuchen ausgeglichen werden. Der hydraulische Versuch dient zum Erfassen des natürlichen Fließverhaltens durch die Kluft, ohne dabei präferenzielle Fließwege erfassen zu wollen. Die Nutzung des gesamten Vorlaufbeckens dient der Beruhigung des Fließverhaltens, sodass das Wasser unter laminaren Strömungsbedingungen in die Kluft eindringt. Nach Medici et al. (2019) bedeutet eine Reynoldszahl zwischen 1 und 10 bei rauen Klüften einen Übergang zwischen laminarem $(<1)$ und turbulentem $(>10)$ Fließen. Der für den Versuchsaufbau anhand von Durchströmungsversuchen bestimmte Wert der Reynoldszahl beläuft sich auf 3 und es wird entsprechend von einem vorwiegend laminaren Fließen ausgegangen.

Bei der hydrochemischen Untersuchung wird das Vorratsbecken erheblich verkleinert, um das benötigte Volumen $\mathrm{zu}$ minimieren und somit Veränderungen im Chemismus deutlicher erkennen zu können. Aufgrund der Größe des Versuchs und den damit einhergehenden hohen Durchflüssen sowie der Unabdingbarkeit synthetisches Grundwasser zu nutzen, werden die Versuchswässer im Kreislauf geführt. Dies wird bei den Auswertungen berücksichtigt und senkt zusätzlich das Risiko, Lösungseffekte in der Kluft auftreten zu lassen, da das genutzte Wasser nicht immer neu ins Gleichgewicht gelangen muss.

Die Gesteinskerne werden für die hydraulischen Versuche mit dem gleichen synthetischen Wasser gesättigt, welches auch für den Durchfluss im Einzelkluftversuch verwendet wird, während für den hydrochemischen Versuch zusätzlich Natriumbromid in die Lösung gegeben wird.

\section{Ergebnisse und Diskussion}

Aus den Untersuchungen an Kernproben gehen die in Tab. 1 aufgeführten Ergebnisse hervor. Der Kalkgehalt von rund 13 Gew. $\%$ befindet sich im unteren Bereich publizierter Werte (Colbach 2005). Sowohl die Messung anhand der archimedischen Dichte als auch über die Gaspyknometrie ergeben vergleichbare Porositäten um 20\%. Bei der archimedischen Dichtebestimmung wurde mit Wasser gearbeitet, wodurch einige Poren und Endporen möglicherweise nicht erfasst wurden und dadurch ein leicht kleinerer Porenanteil als bei der Messung mit Helium festzustellen ist. Colbach (2005) gibt für den Luxemburger Sandstein eine Matrixporosität von 5-35\% an, welche die stark wechselnden Zementgehalte widerspiegelt. Die hier ermittelte Matrixporosität liegt im mittleren Bereich dieser Wertespanne. Sie bestätigt von Struffert (1994) aus Pumpversuchsdaten bestimmte speicherwirksame Porositäten von 19-22\%.

Der durch das Wicking-Experiment an 6 Kernen bestimmte mittlere Porendurchmesser von $10 \pm 2 \mathrm{~nm}$ wirkt sehr klein für einen Sandstein. Ähnliche Werte von anteilig $1 \mathrm{~nm}$ haben z.B. Kashif et al. (2019) an einem paläogenen Sandstein bestimmt. Über Polarisationsmikroskopie eines Dünnschliffes (Abb. 3) sind einige deutlich größere Poren erkennbar, was im Gegensatz zu den vorherigen Messergebnissen steht. Es wird daher vermutet, dass die überschaubare Anzahl an großen Poren im Verhältnis zu etlichen kleinen Poren steht, welche über die Mikroskopie nicht erkennbar sind, und diese feineren Poren den mittleren Porenradius kennzeichnen. Es wird entsprechend eine bipolare Verteilung der Porendurchmesser vermutet. Die Ausdehnung der Poren senkrecht zur betrachteten Ebene in Abb. 3 ist nicht bekannt, wodurch das scheinbare Volumen der Poren zusätzlich überschätzt werden könnte.

Der mittels der Triaxzellenversuche bestimmte hydraulische Durchlässigkeitsbeiwert setzt sich aus zwölf Messungen mit sechs Druckstufen zusammen. Aufgrund der Temperaturempfindlichkeit des Kalk-Kohlensäure-Gleichgewichtes kam es bei selben Druckstufen zu leicht streuenden Ergebnissen. Insgesamt erzeugte die Änderung des

Tab. 1 Ergebnisse der Matrixuntersuchungen am Probenmaterial des Luxemburger Sandsteines

Table 1 Results of matrix studies on the sample material of the Luxembourg Sandstone

\begin{tabular}{ll}
\hline Mittlerer Kalkgehalt & 12,8 Gew.- $\%$ \\
Porosität (Archimedisch) & $19,2 \%$ \\
Porosität (Helium-Gaspyknometrie) & $20,3 \%$ \\
Porendurchmesser & $1 \cdot 10^{-8} \pm 2 \cdot 10^{-9} \mathrm{~m}$ \\
$\begin{array}{l}\text { Hydraulischer Durchlässigkeitsbei- } \\
\text { wert }\end{array}$ & $1,1 \cdot 10^{-6} \pm 2,5 \cdot 10^{-7} \mathrm{~m} / \mathrm{s}$ \\
$\begin{array}{l}\text { Mittlerer effektiver Diffusionskoeffi- } \\
\text { zient }\end{array}$ & $4,4 \cdot 10^{-11} \pm 8,2 \cdot 10^{-12} \mathrm{~m}^{2} / \mathrm{s}$ \\
\end{tabular}


Abb. 3 Aufnahme des Sandsteins unter polarisiertem Licht. In der rechten Hälfte sind die Porenräume mit Rot hervorgehoben

Fig. 3 Image of the sandstone taken under polarized light. In the right half, the pore spaces are highlighted with red color

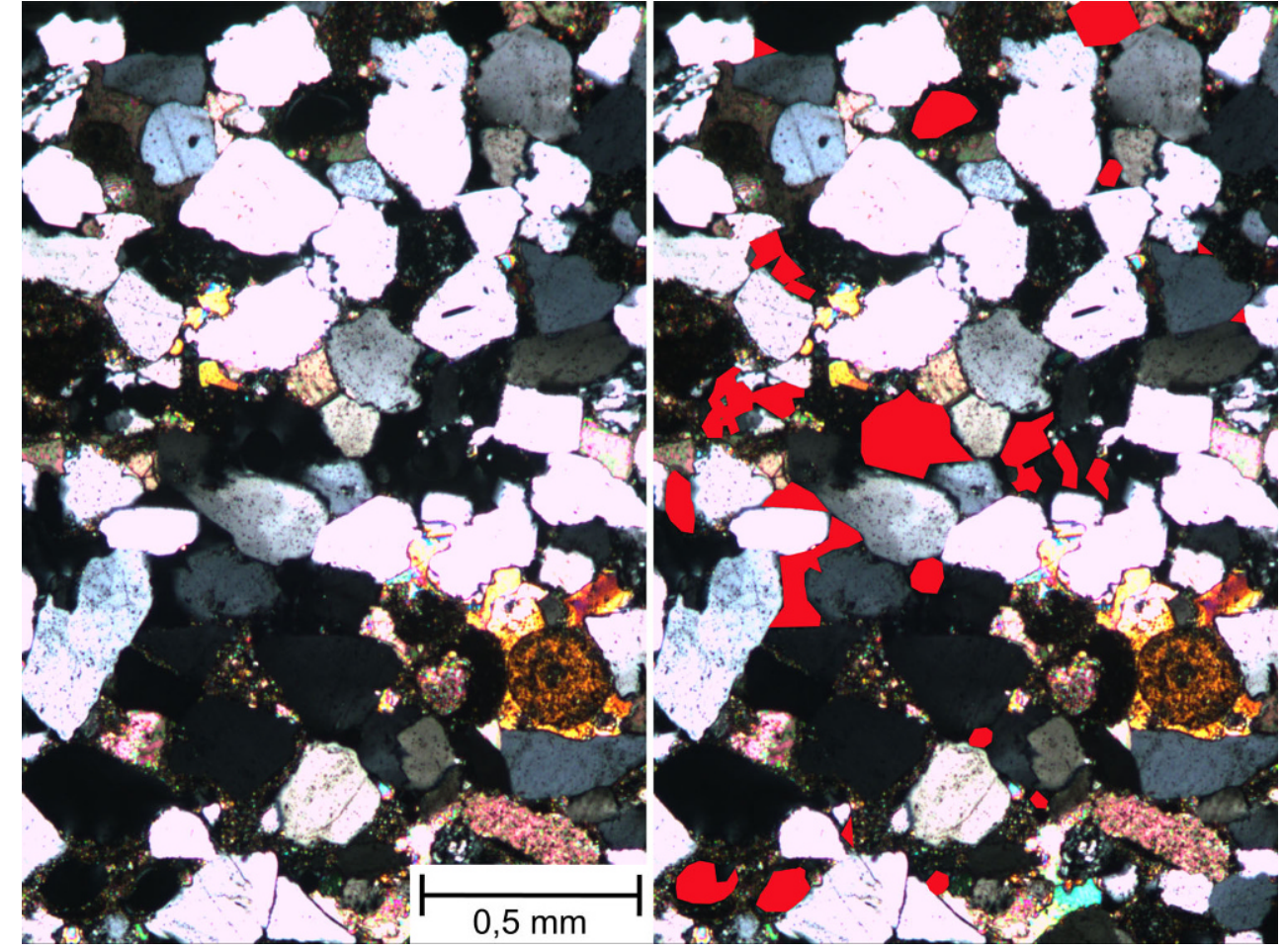

anliegenden Gradienten keine systematischen Änderungen der Messwerte. Der für die Matrix ermittelte Wert von $1,1 \cdot 10^{-6} \pm 0,3 \cdot 10^{-6} \mathrm{~m} / \mathrm{s}$ ist im Vergleich zum publizierten mittleren In-situ-Durchlässigkeitsbeiwert von $5 \cdot 10^{-5} \mathrm{~m} / \mathrm{s}$ (Colbach 2005) kleiner, da letzterer den Einfluss von Klüften beinhaltet.

Anhand von drei Versuchskernen, die drei senkrecht zueinanderstehenden Raumrichtungen entsprechen, wurde der mittlere effektive Diffusionskoeffizient von $4,4 \cdot 10^{-11} \pm$ $0,8 \cdot 10^{-12} \mathrm{~m}^{2} / \mathrm{s}$ bestimmt (s. Tab. 1). Die Unterschiede zwischen den Experimenten in drei Raumrichtungen betragen rund $20 \%$. Entsprechend wird angenommen, dass das untersuchte Gestein isotrop bezüglich der Diffusionsprozesse ist. Der ermittelte Wert des effektiven Diffusionskoeffizienten liegt in der erwarteten Größenordnung für einen Sandstein, z. B. nach Boving und Grathwohl (2001).

Nach dem Erfassen der wichtigsten Parameter der Matrix verbleibt der Einfluss der Kluft auf den hydrochemischen Transport als Untersuchungsziel. Es soll ermittelt werden, inwiefern eine Rückdiffusion in die Kluft stattfindet und ob diese zu den markanten Konzentrationspeaks führen kann, welche in den eingangs beschriebenen Geländeversuchen auftreten können.

Die hydraulische Untersuchung der Kluft ergab bei einem hydraulischen Gradienten von 0,43 einen Durchfluss von $47 \mathrm{ml} / \mathrm{min}$. Über das Cubic Law (z. B. Lomize 1951, Witherspoon et al. 1980) in einer auf die hydraulisch wirksame Apertur für raue, planparallele Kluftflächen abstellenden Gleichung nach Rose (2010) lässt sich vereinfacht die mittlere, hydraulisch wirksame Apertur für die Versuchskluft mit den Dimensionen $0,3 \mathrm{~m} \times 0,3 \mathrm{~m}$ berechnen. Es ergibt sich eine hydraulisch wirksame Apertur von $0,16 \mathrm{~mm}$ bei einer Varianz von 30\%. Der Durchlässigkeitsbeiwert der Kluft berechnet sich nach der Gleichung von Snow (1965) zu $1,9 \cdot 10^{-2} \mathrm{~m} / \mathrm{s}$.

Für die drei ersten Durchgangskurven, in Abb. 4 dargestellt, zeigt sich für die durchgeführten Stop-Flow-Versuche (z.B. Brusseau et al. 1989) die erwartete Verlaufskurve. Der Stop beträgt vor der ersten Verlaufskurve wenige Stunden (Aufgrund der Aufbauphase des Versuchs keine genaue Zeitangabe möglich), zwischen der ersten und zweiten Flow-Phase $18 \mathrm{~h}$ und zwischen der zweiten und dritten Flow-Phase $69 \mathrm{~h}$. Während dieser Stop-Flow-Zeit wurde das Versuchswasser weder gewechselt noch zusätzliches Bromid eingebracht, lediglich das aus der Aufsättigungslösung in der Matrix befindliche Bromid (73,3 mg, über die Porosität und die Konzentration der Aufsättigungslösung berechnet) steht über die Dauer des Versuchs zur Verfügung. Allgemein ist ein Anstieg der Konzentration auf ein erhöhtes Niveau, unmittelbar nach Beendigung des Stops zu beobachten. Dieser Peak fällt mit ähnlicher Geschwindigkeit ab, mit welcher er angestiegen ist, und die Konzentration fällt auf eine Größenordnung wie vor dem Durchströmen ab.

Beim ersten Durchlauf zeigt sich durch das langsam steigende Konzentrationsniveau der Durchgangskurve nach dem Peakdurchgang ein Auswaschen der Matrix, wodurch weiteres Bromid in das Wasser gelangt. Generell entsteht die von Versuch zu Versuch ansteigende Basislinie resp. Ba- 
Abb. 4 Konzentrationsverläufe während der ersten drei Durchspülversuche, Zeitachse in Stunden und Minuten nach Start des Experiments mit Ruhephasen (stop-flow) von ca. $18 \mathrm{~h}$ bzw. ca. $69 \mathrm{~h}$ zwischen den Durchspülungen

Fig. 4 Concentration curves during the first three flushthrough experiments, time axis in hours and minutes after the start of the experiment with rest periods (stop-flow) of approx. $18 \mathrm{~h}$ or approx. $69 \mathrm{~h}$ between flush-throughs

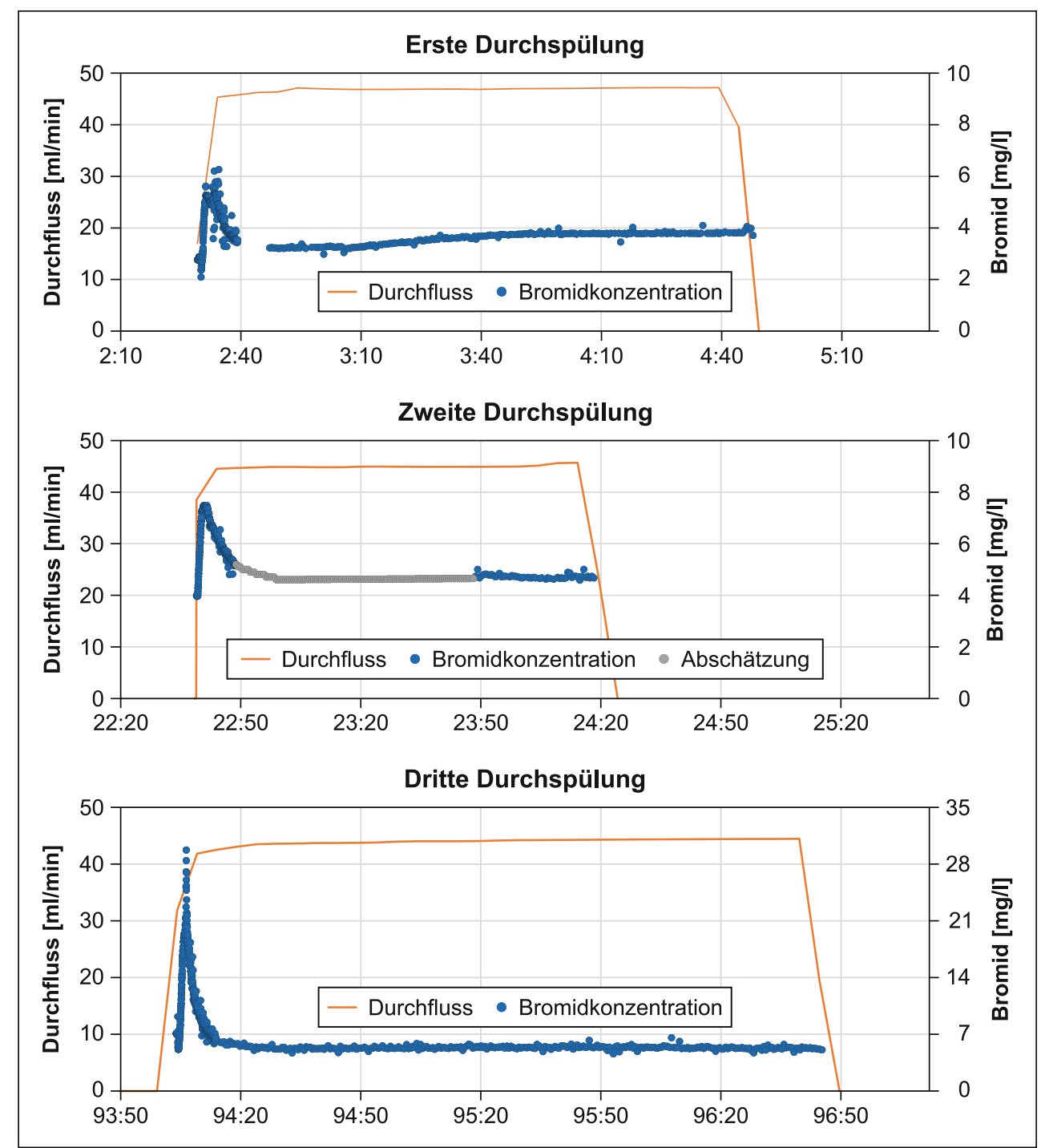

siskonzentration durch die Kreislaufführung des Versuchswassers, sodass jeder Bromidaustrag aus der Matrix diesen Wert anhebt. Für die Versuchsauswertung relevant sind die der Basislinie aufsitzenden Signale.

Bei der zweiten Durchgangskurve wurde ein Teil der Kurve mittels erwartetem Konzentrationsverlauf abgeschätzt, da die Messvorrichtung für diesen Teilabschnitt nicht ordnungsgemäß funktionierte. Da der Konzentrationspeak jedoch vollständig abgebildet wurde und auch die
Verlaufsänderung am Peakende verfügbar ist, wird für die Datenlücke ein quasi linearer Verlauf angenommen. Die dritte Durchgangskurve ähnelt in ihrer Form den beiden vorangegangenen.

Für die Auswertung der Konzentrationskurven wird angenommen, dass das anfängliche Peak-Signal über den reinen Austrag des Kluftwassers, ohne Einfluss von Wasser aus dem Vorrat, entsteht und somit für diese Fracht alleinig die Diffusion aus der Matrix in das Kluftwasser wäh-

Tab. 2 Auswertungsergebnisse der aufgezeigten Stop-Flow-Versuche im Hinblick auf den Stofftransport in der Kluft

Table 2 Evaluation results of the stop-flow tests shown with respect to mass transfer in the fracture

\begin{tabular}{lllllllll}
\hline Durchlauf & $\begin{array}{l}\text { Stop-Zeit } \\
{[\mathrm{h}]}\end{array}$ & $\begin{array}{l}\text { Flow-Zeit } \\
{[\mathrm{h}]}\end{array}$ & $\begin{array}{l}\text { Fracht ge- } \\
\text { samt }[\mathrm{mg}]\end{array}$ & $\begin{array}{l}\text { Fracht Peak } \\
{[\mathrm{mg}]}\end{array}$ & $\begin{array}{l}\text { Fracht } \\
\text { Rest } \\
{[\mathrm{mg}]}\end{array}$ & $\begin{array}{l}\text { Matrixaustrag } \\
{\left[\mathrm{mg} / \mathrm{m}^{2} / \mathrm{s}\right]}\end{array}$ & $\begin{array}{l}\text { Peak Diffu- } \\
\text { sion } \\
{[\mathrm{h}]}\end{array}$ & $\begin{array}{l}\text { Frachtrest Diffu- } \\
\text { sion } \\
{[\mathrm{h}]}\end{array}$ \\
\hline 1 & - & 2,1 & 4,9 & 0,7 & 4,2 & - & - & - \\
2 & ca. 18 & 2,2 & 4,4 & 1,0 & 3,4 & $7,4 \cdot 10^{-5}$ & 20 & 72 \\
3 & ca. 69 & 2,3 & 7,5 & 2,6 & 4,9 & $6,4 \cdot 10^{-5}$ & 63 & 119 \\
\hline
\end{tabular}


rend der Stop-Phase verantwortlich ist. Über den gemessenen Durchfluss und den Konzentrationsverlauf kann so eine Fracht bestimmt werden und über diese zusätzlich zur Stopdauer und der Kluftoberfläche ein Matrixaustrag. Dieser Matrixaustrag ist abhängig von der Konzentrationsdifferenz zwischen dem in der Matrix befindlichen Bromid und dem bereits ausgetragenen im Versuchswasser. Die Stoffmenge in der Matrix lässt sich über die Ausgangsmenge Bromid bei Gesteinsaufsättigung minus dem in dem Versuchswasser befindlichen Bromid berechnen und ermöglicht so eine phasengenaue Bestimmung der Differenz.

Da der erste Durchlauf keine sichere Angabe über die Stop-Dauer besitzt, wird für diesen keine Auswertung vorgenommen.

Aufgrund der Geometrie der Kluft und der damit verbundenen präferenziellen Fließwege, wird nicht die gesamte Kluftfläche durchströmt, und der ermittelte Wert gibt nur einen Mittelwert über die planare Fläche wieder.

Durch den leichten Anstieg der Basiskurve bei jedem Flow-Versuch, zeigt sich ein zusätzlicher Austrag von Bromid, der anhand der Start- und Endkonzentrationen bestimmt werden kann. Vergleicht man in Tab. 2 die beobachteten Frachtausträge mit den rechnerisch bestimmten Zeiten, die eine reine Diffusion (berechnet mittels des an Kernen bestimmten effektiven Diffusionskoeffizienten) benötigen würde, zeigt sich, dass der Austrag aus der Matrix in die Kluft für die Stop-Dauer über Diffusion möglich ist, jedoch die Flow-Dauer unzureichend ist.

Es ist entsprechend anzunehmen, dass der Matrixbereich im Umfeld der Kluft bei auftretender Strömung ebenfalls durchflossen wird und mit einer schnellen Komponente zusätzlich zum Austrag von Bromid beiträgt. Zum Erreichen der ausgewaschenen Bromidmasse, muss zusätzlich zur Kluft ein Matrixbereich von $0,7 \mathrm{~cm}$ je Kluftseite durchströmt werden. Dies unter der Annahme einer homogenen Konzentrations- und Porenraumverteilung in der Matrix. Für den Stofftransport im Luxemburger Sandstein ist demnach nicht der reine Transport in der Kluft sowie/oder der Matrix verantwortlich, sondern ebenfalls eine Mischform durch die Durchströmung des Randbereiches zwischen Gesteinsmatrix und den Klüften. Der im Feld beobachtete peakartige Stoffaustrag lässt sich demnach auf das Zusammenspiel zwischen Rückdiffusion aus der Matrix in das Kluftwasser während vorherigen, strömungsschwachen Phasen und die Durchströmung poröser Matrix-KluftRandzonen unmittelbar während strömungsintensiven Phasen zurückführen.

\section{Ausblick}

Anhand der erfolgten Versuche zeigt sich, dass der Stofftransport im Luxemburger Sandstein erheblich durch die
Interaktion zwischen Matrix und Kluft gesteuert wird. Sowohl über Diffusion als auch über die Durchströmung der kluftnahen Matrix werden transportierte Stoffe in die maßgeblichen hydraulischen Transportwege abgegeben und im Umkehrschluss auch in der Matrix retardiert.

Da die Durchführung eines Einzelkluftversuchs nicht für jede Ausprägung der Sandsteinfazies möglich ist, aber dennoch für die Bewirtschaftung des Aquifers wichtige Erkenntnisse bergen könnte, werden in einem nächsten Schritt die gewonnenen Parameter der Matrix sowie die Ergebnisse der Laborversuche genutzt, um eine Modellierung mittels des frei zugänglichen 3D-Simulationsprogrammes PHAST für Strömung, Stofftransport und geochemische Reaktionen (Parkhurst et al. 2010) zu kalibrieren. Gelingt dies, werden zur Modellierung des Stofftransportes in den geklüfteten Gesteinen lediglich die Matrixparameter benötigt. Dies erfolgt unter der Annahme, dass die Klüfte in der gesamten Fazies des Luxemburger Sandsteines ähnliche Eigenschaften besitzen und lediglich die inerten Gesteinseigenschaften Unterschiede aufweisen.

Funding Open Access funding enabled and organized by Projekt DEAL.

Open Access Dieser Artikel wird unter der Creative Commons Namensnennung 4.0 International Lizenz veröffentlicht, welche die Nutzung, Vervielfältigung, Bearbeitung, Verbreitung und Wiedergabe in jeglichem Medium und Format erlaubt, sofern Sie den/die ursprünglichen Autor(en) und die Quelle ordnungsgemäß nennen, einen Link zur Creative Commons Lizenz beifügen und angeben, ob Änderungen vorgenommen wurden.

Die in diesem Artikel enthaltenen Bilder und sonstiges Drittmaterial unterliegen ebenfalls der genannten Creative Commons Lizenz, sofern sich aus der Abbildungslegende nichts anderes ergibt. Sofern das betreffende Material nicht unter der genannten Creative Commons Lizenz steht und die betreffende Handlung nicht nach gesetzlichen Vorschriften erlaubt ist, ist für die oben aufgeführten Weiterverwendungen des Materials die Einwilligung des jeweiligen Rechteinhabers einzuholen.

Weitere Details zur Lizenz entnehmen Sie bitte der Lizenzinformation auf http://creativecommons.org/licenses/by/4.0/deed.de.

\section{Literatur}

AGE: Eist Waasser Bd. 118. Administration de la Gestion de l'eau, Luxemburg (2013)

AGE: Entwurf des dritten Bewirtschaftungsplans für die luxemburgischen Anteile an den internationalen Flussgebietseinheiten Rhein und Maas (2021-2027). Administration de la Gestion de l'eau, Luxemburg (2021)

Berre, I., Doster, F., Keilegavlen, E.: Flow in fractured porous media: A Review of conceptual models and discretization approaches. Transp Porous Med 130, 215-236 (2019)

Bodin, J., Delay, F., de Marsily, G.: Solute transport in a single fracture with negligible matrix permeability $: 1$. fundamental mechanisms. Hydrogeol J 11, 418-433 (2003)

de Borst, R.: Fluid flow in fractured and fracturing porous media: A unified view. Mech. Res. Comminucations 80, 47-57 (2017) 
Boving, T.B., Grathwohl, P.: Tracer diffusion coefficient in sedimentary rocks: correlation to porosity and hydraulic conductivity. J. Contam. Hydrol. 53, 85-100 (2001)

Brusseau, M.L., Rao, P.S.C., Jessup, R.E., Davidson, J.M.: Flow interruption: a method for investigating sorption nonequilibrium. J. Contam. Hydrol. 4, 223-240 (1989)

Colbach, R.: Overview of the geology of the Luxembourg Sandstone(s). Ferrantia 44, 155-160 (2005)

D'Angelo, P., Migliorati, V., Guidoni, L.: Hydration properties of the bromide aqua ion: The interplay of first principle and classical molecular dynamics, and x-ray absorption spectroscopy. Inorg. Chem. 49, 4224-4231 (2010)

Dittrich, D.: Erläuterungen zur Geologischen Karte von Luxemburg. Service Géologique du Luxembourg, Luxembourg (1984). Blatt Nr. 8 Mersch

Feenstra, S., Cherry, J.A., Sucicky, E.A., Haq, Z.: Matrix diffusion effects on contaminant migration from an injection well in fractured sandstone. Groundwater 22(3), 307-316 (1984)

Haddad, A.S., Hassanzadeh, H., Abedi, J.: Advective-diffusive mass transfer in fractured porous media with variable rock matrix block size. J. Contam. Hydrol. 133, 94-107 (2012)

Karay, G., Hajnal, G.: Modelling of groundwater flow in fractured rocks. Procedia Environ. Sci. 25, 142-149 (2015)

Kashif, M., Cao, Y., Yuan, G., Asif, M., Javed, K., Mendez, J.N., Khan, D., Miruo, L.: Pore size distribution, their geometry and connectivity in deeply buried Paleogene Es1 sandstone reservoir, Nanpu Sag, East China. Petroleum Sci. 16, 981-1000 (2019)

Kausch, B., Maquil, R.: Landscapes and landforms of the Luxembourg sandstone, grand-duchy of Luxembourg. In: Demoulin, A. (Hrsg.) Landscapes and landforms of Belgium and Luxembourg, S. 43-62. Springer, Cham (2018)

Langguth, H.-R., Voigt, R.: Hydrogeologische Methoden. Springer, Berlin, Heidelberg (2004)

Leibundgut, C., Maloszewski, P., Külls, C.: Tracers in hydrology. John Wiley \& Sons, Chichester (2009)

Lomize, G.M.: Fluid flow in fractured rocks. Gosenergoizdat, Moscow (1951). in Russisch

Medici, G., West, L.J., Banwart, S.A.: Groundwater flow velocities in a fractured carbonate aquifer-type: Implications for contaminant transport. J. Contam. Hydrogeol. 222, 1-16 (2019)

Meus, P., Willems, L.: Tracer tests to infer the drainage of the multiple porosity aquifer of Luxembourg Sandstone (Grand-Duchy of Luxembourg): implications for drinking water protection. Hydrogeol J 29, 461-480 (2021)
Parkhurst, D.L., Kipp, K.L., Charlton, S.R.: PHAST Version 2 - A Program for Simulating Groundwater Flow, Solute Transport, and Multicomponent Geochemical Reactions. U.S. Geological Survey, Denver (2010)

Reisinger, T.: Stofftransport von Spurenelementen im Tiefengrundwasser in Sandstein-Kluftaquiferen. RT Geologie, Aachen (2020)

Rose, T.A.: Zur Strömungssimulation in Einzelklüften: Gegenüberstellung von numerischen Methoden und Experimenten. Mitteilungen des Lehrstuhls und Instituts für Wasserbau und Wasserwirtschaft der RWTH Aachen. Shaker, Düren (2010)

Schubert, A.C.: Grundwasserressourcen im östlichen Eischtal in Luxemburg - Quantifizierung des verfügbaren Grundwassers und die Systematik seiner Quelllagen. RWTH Aachen University, Aachen (2015). Bachelorarbeit

Snow, D.T.: A parallel plate model of fractured permeable media (1965). Dissertation an der University of California

Struffert, F.-J.: Hydrogeologische Detailuntersuchung im Rahmen der „Solution de Rechange“ (Ersatzlösung) in Luxemburg. Veröffentlichungen des Luxemburger Geologischen Dienstes, Bd. 18. Service Géologique du Luxembourg, Luxembourg (1994)

Tang, D.H., Frind, E.O., Sudicky, E.A.: Contaminant transport in fractured porous media: analytical solution for a single fracture. Water Resour. Res. 17(3), 555-564 (1981)

Thielen, C.: Untersuchungen des Einflusses einer Brunnenförderung auf Quellschüttung und die Fluss-Grundwasser-Interaktion im Eischtal bei Koerich (Luxemburg) als Grundlage für ein Monitoring. RWTH Aachen University, Aachen (2017). Masterarbeit

Weber, C., Stanjek, H.: Development of diffuse double layers in column-wicking experiements: Implications for $\mathrm{pH}$-dependent contact angles on quarz. J. Colloid. Interface. Sci. 387(1), 270-274 (2012)

Weede, M., Hötzl, H.: Strömung und Transport in einer natürlichen Einzelkluft in poröser Matrix - Experimente und Modellierung. Grundwasser 10(3), 137-145 (2005)

Witherspoon, P.A., Wang, J.S.Y., Iwai, K., Gale, J.E.: Validity of cubic law for fluid flow in a deformable rock fracture. Water Resour. Res. 16, 1016-1024 (1980)

Hinweis des Verlags Der Verlag bleibt in Hinblick auf geografische Zuordnungen und Gebietsbezeichnungen in veröffentlichten Karten und Institutsadressen neutral. 